\title{
EXPLICIT LOWER BOUNDS FOR RATIONAL APPROXIMATION TO ALGEBRAIC NUMBERS
}

\author{
MICHAEL A. BENNETT \\ [Received 24 November 1995-Revised 20 August 1996]
}

\section{Introduction}

Lower bounds for rational approximation to algebraic numbers have been studied for many years, both for their intrinsic interest and for their applications to Diophantine equations. At one end of the spectrum, we have the weak bound due to Liouville who showed that if $\theta$ is an algebraic number of degree $n$, then

$$
\left|\theta-\frac{p}{q}\right|>c(\theta) q^{-n}
$$

for non-zero integers $p$ and $q$. At the other end, we have the famous inequality of Roth [19] to the effect that, for $\varepsilon>0$,

$$
\left|\theta-\frac{p}{q}\right|>c(\theta, \varepsilon) q^{-2-\varepsilon}
$$

again for any non-zero $p$ and $q$. Unfortunately, while the first of these bounds is effective in that the constant $c(\theta)$ is computable from Liouville's proof, Roth's theorem is not. Efforts to produce effective improvements upon Liouville's bound have centred about three lines of attack: the theory of linear forms in logarithms (see for example, $[\mathbf{3}, \mathbf{4}, \mathbf{1 3}]$ ), the Thue principle (see $[\mathbf{8}, \mathbf{9}]$ ) and the method of Baker and Siegel, using rational function approximation (see $[1,2,5,6,11,12,14,17])$. While the first of these techniques is indisputably more general, we restrict our attention in this paper to the last, which is characterized by attractive and strong bounds in special settings. In [1] and [2], via Padé approximation to the binomial function $(1-z)^{v}$, Baker was able to obtain effective improvements upon Liouville's theorem for restricted classes of algebraic numbers. In particular, he showed that

$$
\left|\sqrt[3]{2}-\frac{p}{q}\right|>10^{-6} q^{-2 \cdot 955}
$$

for all positive integers $p$ and $q$. This implies that solutions $(x, y)$ to the Diophantine equation

$$
x^{3}-2 y^{3}=u
$$

satisfy

$$
\max \{|x|,|y|\} \leqslant\left(3.10^{5} \cdot|u|\right)^{23} .
$$


These results were subsequently sharpened by Chudnovsky [11], who improved inequality (1) to

$$
\left|\sqrt[3]{2}-\frac{p}{q}\right|>c q^{-2 \cdot 42971}
$$

for $p$ and $q$ positive integers and $c$ some effectively computable constant. The value of $c$, however, was left undetermined and, for technical reasons, is in fact extremely difficult to compute. In [6], though, the author used a variation upon Chudnovsky's approach to produce a completely explicit version of (2), of close to the same strength, namely that

$$
\left|\sqrt[3]{2}-\frac{p}{q}\right|>\frac{1}{4} q^{-2 \cdot 47}
$$

for integers $p$ and $q(q \neq 0)$. This implies that

$$
\left|x^{3}-2 y^{3}\right| \geqslant \sqrt{x}
$$

for all non-negative integers $x$ and $y$. The essential ingredient of this approach, as compared to that of [11] and [12], is that certain required estimates for primes in arithmetic progressions may be replaced by sharper estimates over all primes in given intervals. While, in the latter paper, we considered only examples of cubic irrationalities, here we turn our attention to algebraic numbers of higher degree. Defining, for positive integers $a$ and $n$,

$$
\kappa(a, n)=\prod_{p \mid n} p^{\max \left\{\operatorname{ord}_{p}(n / a)+1 /(p-1), 0\right\}}
$$

where the product is over prime $p$, we prove

THEOREM 1.1. For integer $n$, define the constant $c_{1}(n)$ as follows:

\begin{tabular}{ll|ll|ll|ll|ll}
\hline$n$ & $c_{1}(n)$ & $n$ & $c_{1}(n)$ & $n$ & $c_{1}(n)$ & $n$ & $c_{1}(n)$ & $n$ & $c_{1}(n)$ \\
\hline 3 & 2.03 & 11 & 1.67 & 23 & 1.53 & 41 & 1.45 & 59 & 1.40 \\
4 & 1.62 & 13 & 1.65 & 29 & 1.51 & 43 & 1.43 & 61 & 1.39 \\
5 & 1.84 & 17 & 1.58 & 31 & 1.51 & 47 & 1.44 & 67 & 1.38 \\
7 & 1.76 & 19 & 1.56 & 37 & 1.46 & 53 & 1.40 & 71 & 1.36 \\
\hline
\end{tabular}

Suppose that $a, N, s$ and $n$ are integers with $1 \leqslant a<N, 1 \leqslant s<\frac{1}{2} n,(s, n)=1$ and $n$ occurring in the above table. If, further, we have

$$
(\sqrt{N}+\sqrt{N+a})^{2(n-2)}>a^{2(n-1)}\left(\frac{\kappa(a, n)}{c_{1}(n)}\right)^{n},
$$

then we can conclude that

$$
\left|\left(1+\frac{a}{N}\right)^{s / n}-\frac{p}{q}\right|>N^{-1}\left(10^{10} q\right)^{-\lambda}
$$

with

$$
\lambda=1+\frac{\log \left(\left(\kappa(a, n) / c_{1}(n)\right)(\sqrt{N}+\sqrt{N+a})^{2}\right)}{\log \left(\left(c_{1}(n) / a^{2} \kappa(a, n)\right)(\sqrt{N}+\sqrt{N+a})^{2}\right)} .
$$


Through application of the above theorem, we may derive explicit irrationality measures for certain $\sqrt[n]{m}$ with $m \in \mathbb{Z}$. For algebraic numbers of this form, generating distinct number fields and satisfying $2 \leqslant m \leqslant 50$, we have

COROLlaRY 1.2. If $p$ and $q$ are positive integers, then we have

$$
\left|\theta-\frac{p}{q}\right|>c(\theta) q^{-\lambda(\theta)}
$$

where we may take $\theta, c(\theta)$ and $\lambda(\theta)$ as in Table 1.

TABLE 1.

\begin{tabular}{|c|c|c|c|c|c|c|c|c|}
\hline$\theta$ & $c(\theta)$ & $\lambda(\theta)$ & $\theta$ & $c(\theta)$ & $\lambda(\theta)$ & $\theta$ & $c(\theta)$ & $\lambda(\theta)$ \\
\hline$\sqrt[4]{5}$ & 0.03 & 2.77 & $\sqrt[5]{18}$ & $0 \cdot 21$ & $4 \cdot 29$ & $\sqrt[7]{10}$ & 0.38 & $5 \cdot 17$ \\
\hline$\sqrt[4]{14}$ & 0.06 & 3.78 & $\sqrt[5]{22}$ & $0 \cdot 14$ & 4.91 & $\sqrt[7]{11}$ & 0.40 & 3.34 \\
\hline$\sqrt[4]{15}$ & 0.03 & $3 \cdot 27$ & $\sqrt[5]{28}$ & 0.05 & 3.41 & $\sqrt[7]{12}$ & 0.42 & 3.88 \\
\hline$\sqrt[4]{17}$ & 0.03 & $3 \cdot 24$ & $\sqrt[5]{30}$ & 0.02 & 3.04 & $\sqrt[7]{13}$ & 0.44 & 4.91 \\
\hline$\sqrt[4]{18}$ & 0.05 & 3.67 & $\sqrt[5]{31}$ & $0 \cdot 01$ & $2 \cdot 83$ & $\sqrt[7]{17}$ & 0.03 & $5 \cdot 20$ \\
\hline$\sqrt[4]{37}$ & 0.33 & $3 \cdot 34$ & $\sqrt[5]{33}$ & 0.01 & $2 \cdot 82$ & $\sqrt[7]{23}$ & 0.43 & 6.03 \\
\hline$\sqrt[4]{39}$ & 0.005 & 2.52 & $\sqrt[5]{34}$ & 0.02 & 3.02 & $\sqrt[7]{45}$ & $0 \cdot 27$ & $5 \cdot 10$ \\
\hline$\sqrt[5]{3}$ & $0 \cdot 24$ & $3 \cdot 61$ & $\sqrt[5]{37}$ & 0.05 & 3.48 & $\sqrt[11]{48}$ & 0.42 & 5.05 \\
\hline$\sqrt[5]{6}$ & 0.43 & $3 \cdot 33$ & $\sqrt[5]{39}$ & 0.08 & 2.91 & $\sqrt[13]{6}$ & $0 \cdot 14$ & 4.22 \\
\hline$\sqrt[5]{10}$ & 0.41 & 3.92 & $\sqrt[5]{40}$ & 0.09 & 3.90 & $\sqrt[13]{20}$ & 0.25 & 5.87 \\
\hline$\sqrt[5]{11}$ & $0 \cdot 38$ & $4 \cdot 23$ & $\sqrt[5]{42}$ & $0 \cdot 11$ & 4.19 & $\sqrt[17]{50}$ & 0.25 & 6.96 \\
\hline$\sqrt[5]{15}$ & $0 \cdot 28$ & $4 \cdot 27$ & $\sqrt[7]{5}$ & $0 \cdot 25$ & 4.43 & & & \\
\hline
\end{tabular}

For a forthcoming paper [7] on Diophantine equations, we require a specialization of Theorem 1.1 to the cases where $a=s=1$, valid for all $n \geqslant 3$. Defining

$$
\mu_{n}=\prod_{p \mid n} p^{1 /(p-1)},
$$

we have

THEOREM 1.3. If $n$ and $N$ are positive integers with $n \geqslant 3$ and

$$
(\sqrt{N}+\sqrt{N+1})^{2(n-2)}>\left(n \mu_{n}\right)^{n}
$$

then

$$
\left|\sqrt[n]{1+\frac{1}{N}}-\frac{p}{q}\right|>\left(8 n \mu_{n} N\right)^{-1} q^{-\lambda}
$$

with

$$
\lambda=1+\frac{\log \left(n \mu_{n}(\sqrt{N}+\sqrt{N+1})^{2}\right)}{\log \left(\left(1 / n \mu_{n}\right)(\sqrt{N}+\sqrt{N+1})^{2}\right)} .
$$


Before proceeding, we note that the restriction in Theorem 1.1 that $1 \leqslant s<\frac{1}{2} n$ is unimportant since a non-trivial irrationality measure for $(1+a / N)^{s / n}$ implies one for $(1+a / N)^{1-s / n}$ (the latter being a rational multiple of the reciprocal of the former). We similarly exclude non-prime $n \geqslant 6$ from Theorem 1.1 , since an inequality of the form

$$
\left|\left(1+\frac{a}{N}\right)^{s / n}-\frac{p^{k}}{q^{k}}\right|<c_{1}\left(q^{k}\right)^{-\lambda}
$$

with $\lambda<n$, implies

$$
\left|\left(1+\frac{a}{N}\right)^{s / n k}-\frac{p}{q}\right|<c_{2} q^{-k \lambda} .
$$

For this reason, in Corollary 1.2, we do not list irrationality measures for numbers of the form (for example) $\sqrt[6]{m}$. Applying our machinery directly with $n=6$ fails to yield new examples with $m \leqslant 100$ (though the related measures are on occasion improved). Bounds for rational approximation to certain $\sqrt[3]{m}$ are given in [6] and will not be repeated here, though we mention that Theorem 1.1 slightly sharpens the corresponding result in [6]. This implies, for instance, an improvement of (3) to

$$
\left|\sqrt[3]{2}-\frac{p}{q}\right|>\frac{1}{4} q^{-2 \cdot 45}
$$

for all positive $p$ and $q$.

\section{Technical preliminaries}

To deduce the aforementioned results, we appeal to the following lemma, obtained from the proof of Lemma 2.1 of [17]:

LEMMA 2.1. Suppose $\theta$ is real and that there exist positive real numbers $c, d$, $C$ and $D(D>1)$ such that for each positive integer $k$ with $k \geqslant k_{0}\left(k_{0} \in \mathbb{N}\right)$, we can find integers $p_{\text {lmk }}(0 \leqslant l, m \leqslant 1)$ with non-zero determinant,

$$
\left|p_{l m k}\right| \leqslant c C^{k} \quad(0 \leqslant l, m \leqslant 1)
$$

and

$$
\left|p_{l 0 k}+p_{l 1 k} \theta\right| \leqslant d D^{-k} \quad(0 \leqslant l \leqslant 1) .
$$

Then, if $t$ is any real number with $t>1$, we may conclude that

$$
\left|\theta-\frac{p}{q}\right|>\left(t c C\left(\max \left\{1, \frac{t d}{t-1}\right\}\right)^{\log (C) / \log (D)}\right)^{-1} q^{-1-\log (C) / \log (D)}
$$

for all positive integers $p$ and $q$ with $q \geqslant D^{k_{0}-1}$.

As in [6], to apply this result we require a sequence of good rational approximations to numbers of the form $(1+a / N)^{s / n}$. To find these, we construct the diagonal Padé approximants to $(1+a x)^{s / n}$, from the contour integral

$$
I_{l}(x)=\frac{1}{2 \pi i} \int_{\gamma} \frac{(1+z x)^{k+s / n}}{(z-l a)(z(z-a))^{k}} d z \quad(0 \leqslant l \leqslant 1),
$$


where $|x|<1 / a$ and $\gamma$ is a closed counter-clockwise contour enclosing 0 and $a$. Lemma 3.3 of [17] implies, then, that

$$
I_{l}(x)=p_{l 0}(x)+(1+a x)^{s / n} p_{l 1}(x) \quad(0 \leqslant l \leqslant 1),
$$

where

$$
p_{l m}(x)=a^{-2 k} \sum_{r=0}^{k_{l m}}(-1)^{m r+k_{l m}}\left(\begin{array}{c}
k+s / n \\
r
\end{array}\right)\left(\begin{array}{c}
2 k-r-1 \\
k_{l m}-r
\end{array}\right)(a x)^{r}(1+a x m)^{k-r}
$$

with $k_{l m}=k-1+\delta_{l m}$ and $\delta_{l m}$ the Kronecker delta $(0 \leqslant l, m \leqslant 1)$. We prove

LEMMA 2.2. Suppose that $a, N, s$ and $n$ are positive integers satisfying the hypotheses of either Theorem 1.1 or Theorem 1.3 and that $0 \leqslant l, m \leqslant 1$ and $k \geqslant 1$. Then

$$
\left|I_{l}(1 / N)\right| \leqslant \frac{5 \cdot 2 s}{n}\left(N(\sqrt{N}+\sqrt{N+a})^{2}\right)^{-k}
$$

and

$$
\left|p_{l m}(1 / N)\right| \leqslant 1 \cdot 3\left(\frac{(\sqrt{N}+\sqrt{N+a})^{2}}{a^{2} N}\right)^{k}
$$

Proof. (i) For $n=3$, the result follows from Lemma 2.1 of [6], upon noting that

$$
(\sqrt{N}+\sqrt{N+a})^{2}>a^{4}\left(\frac{\kappa(a, 3)}{2 \cdot 03}\right)^{3}
$$

implies that $N \geqslant 4 a$ (since $N$ and $a$ are integral). We may therefore assume that $n \geqslant 4$. Suppose that $a, n$ and $N$ satisfy

$$
(\sqrt{N}+\sqrt{N+a})^{2(n-2)}>a^{2(n-1)}\left(\frac{\kappa(a, n)}{c}\right)^{n},
$$

where we will take $c=c_{1}(n)$ or $c=1$ as appropriate. If we fix $\delta=N / a$, this therefore yields

$$
2 \delta+1+2 \sqrt{\delta(\delta+1)}>\left(\frac{a \kappa(a, n)}{c}\right)^{n /(n-2)} \geqslant\left(\frac{n \mu_{n}}{c}\right)^{n /(n-2)} .
$$

From the proof of Lemma 3.2 of [5],

$$
\left|I_{l}(1 / N)\right|=\frac{\left|1-e^{2 \pi i s / n}\right|}{2 \pi} N^{-2 k} \int_{0}^{\infty} \frac{x^{k+s / n} d x}{(x+1+a l / N)((x+1)(x+1+a / N))^{k}}
$$

whence, for $k \geqslant 4$, we can use the inequalities

$$
\left|1-e^{2 \pi i s / n}\right|<\frac{2 \pi s}{n} \text { and } \frac{x^{s / n}}{x+1}<1
$$

(where the latter is valid provided $x>0$ ) to deduce that

$$
\left|I_{l}(1 / N)\right|<\frac{s}{n} N^{-2 k} \int_{0}^{\infty}\left(\frac{x}{(x+1)(x+1+a / N)}\right)^{k} d x .
$$


Write this last integral as

$$
\int_{0}^{4}\left(\frac{x}{(x+1)(x+1+a / N)}\right)^{k} d x+\int_{4}^{\infty}\left(\frac{x}{(x+1)(x+1+a / N)}\right)^{k} d x
$$

By calculus, the first of these is bounded by

$$
4 N^{k}(\sqrt{N}+\sqrt{N+a})^{-2 k},
$$

while the second is bounded above by

$$
\int_{4}^{\infty}\left(\frac{x}{(x+1)^{2}}\right)^{k} d x<5^{-k}
$$

for $k \geqslant 4$. From (4), we find that $\delta>2$ for each $n \geqslant 4$ with either choice of $c=c_{1}(n)$ or $c=1$. This implies that

$$
\frac{(\sqrt{N}+\sqrt{N+a})^{2}}{N}<5
$$

in all cases under consideration, so that

$$
\int_{0}^{\infty}\left(\frac{x}{(x+1)(x+1+a / N)}\right)^{k} d x<5\left(\frac{(\sqrt{N}+\sqrt{N+a})^{2}}{N}\right)^{-k},
$$

which gives the desired result.

If $k=1$, then we note that

$$
\int_{0}^{\infty} \frac{x^{1+s / n} d x}{(x+1)^{2}(x+1+a / N)}<\int_{0}^{\infty} \frac{x^{1+s / n} d x}{(x+1)^{3}}
$$

which equals

$$
\frac{n \Gamma(1-s / n) \Gamma(3+s / n)}{2(2 n+s)} .
$$

Since $1 \leqslant s<\frac{1}{2} n$, this is less than $\frac{3}{8} \pi$ and hence less than $1 \cdot 1781$. Now, from (4), we have $\delta>5$ for $n=4$ and $n \geqslant 23$ (assuming $c=c_{1}(n)$ ) and for all $n \geqslant 4$ (with $c=1$ ). This implies that

$$
\frac{(\sqrt{N}+\sqrt{N+a})^{2}}{N}<4 \cdot 4
$$

and hence that

$$
\left|I_{l}(1 / N)\right|<\frac{5 \cdot 2 s}{n}\left(N(\sqrt{N}+\sqrt{N+a})^{2}\right)^{-1} .
$$

For $n=5,7,11,13$ and 17 , we compute

$$
\int_{0}^{\infty} \frac{x^{1+s / n} d x}{(x+1)^{3}}
$$

for each $1 \leqslant s<\frac{1}{2} n$, find explicit values for $\left|1-e^{2 \pi i s / n}\right|$ and use (4) to derive lower bounds upon the related $\delta$ 's. In all cases, we verify that (5) is satisfied.

Finally, if $2 \leqslant k \leqslant 3$, we use the inequality

$$
\int_{0}^{\infty} \frac{x^{k+s / n} d x}{(x+1)((x+1)(x+1+a / N))^{k}}<\int_{0}^{1} \frac{x^{k} d x}{(x+1)^{2 k+1}}+\int_{1}^{\infty} \frac{x^{k+1 / 2} d x}{(x+1)^{2 k+1}}
$$


and check that the right-hand side is bounded above by $5 \cdot 2 \times 5^{-k}$ in both cases, whence

$$
\left|I_{l}(1 / N)\right|<\frac{5 \cdot 2 s}{n}\left(N(\sqrt{N}+\sqrt{N+a})^{2}\right)^{-k}
$$

for all $k$, as desired.

(ii) Again, we may assume (via Lemma 2.3 of [6]) that $n \geqslant 4$. Repeating the arguments from that lemma, we have

$$
\left|p_{l m}(1 / N)\right| \leqslant \sqrt{\frac{N+a}{N}}\left(2-\sqrt{\frac{N}{N+a}}\right)^{s / n}\left(\frac{(\sqrt{N}+\sqrt{N+a})^{2}}{a^{2} N}\right)^{k} .
$$

From (4), in all cases except when $n=5,7$ or 11 and $c=c_{1}(n)$, we have $\delta>3$, whence

$$
\sqrt{\frac{N+a}{N}}\left(2-\sqrt{\frac{N}{N+a}}\right)^{s / n}<\sqrt{\frac{N+a}{N}}\left(2-\sqrt{\frac{N}{N+a}}\right)^{1 / 2}<1 \cdot 3 .
$$

If $n=5,7$ or 11 and $c=c_{1}(n)$, then we have $\delta$ bounded below by $\frac{11}{5}, \frac{16}{7}$ or $\frac{31}{11}$, respectively. It follows that

$$
\sqrt{\frac{N+a}{N}}\left(2-\sqrt{\frac{N}{N+a}}\right)^{s / n}<\sqrt{\frac{N+a}{N}}\left(2-\sqrt{\frac{N}{N+a}}\right)^{(n-1) / 2 n}<1 \cdot 3
$$

in each remaining case, which completes the proof.

\section{Coefficients of the $p_{l m}(1 / N)$}

As in $[5,6,11,12,14]$, we refine more classical estimates through a careful analysis of the common factors present in the numerators of the (rational) coefficients of the polynomials $p_{l m}(x)$. Lemma 4.2 of [11] implies that if $n, s, r$, $j$ and $k$ are non-negative integers with $1 \leqslant s<n$ and $k \geqslant 1$, then

$$
n^{j} \prod_{p \mid n} p^{[j /(p-1)]}\left(\begin{array}{c}
k+s / n \\
r
\end{array}\right)
$$

is an integer for each $0 \leqslant r \leqslant j$ (where $[x]$ denotes the greatest integer not greater than $x$ ). We may thus define

$$
G(j)=\operatorname{gcd}\left\{n^{j} \prod_{p \mid n} p^{[j /(p-1]}\left(\begin{array}{c}
k+s / n \\
r
\end{array}\right)\left(\begin{array}{c}
2 k-r-1 \\
j-r
\end{array}\right): r=0,1, \ldots, j\right\}
$$

and take

$$
G_{k}=\operatorname{gcd}\{G(k), G(k-1)\},
$$

suppressing dependence on $s$ and $n$. We prove

LEMMA 3.1. For $n$ and $c_{1}(n)$ appearing in Theorem 1.1 and $k$ and $s$ positive integers with $1 \leqslant s<\frac{1}{2} n$, we have

except for $n=4$, where

$$
G_{k}>e^{-16} c_{1}(n)^{k}
$$

$$
G_{k}>e^{-20} c_{1}(4)^{k} \text {. }
$$

To obtain this result, we require 
Lemma 3.2. Suppose that $k \geqslant 1$ and let $s$ and $n$ be integers with $n \geqslant 3$, $1 \leqslant s<\frac{1}{2} n$ and $(s, n)=1$. If $p$ is a prime with $(p, n)=1$ and $q$ is chosen such that $1 \leqslant q<\frac{1}{2} n$ and

$$
p q \equiv \pm s \quad(\bmod n)
$$

then if

$$
p \in \bigcup_{j=1}^{[\sqrt{k / n}]}\left[\frac{k+1}{j}, \frac{n k-n-1}{n j-q}\right],
$$

we have that $p$ divides $G_{k}$.

Proof (of Lemma 3.2). This is essentially just a slight generalization of Lemma 3.1 of [6]. Condition (6) implies that $(p, k)=1, p>\sqrt{n k+s}$ and $\{(k-1) / p\}>(n-q) / n$ which together are sufficient to guarantee that one of $\operatorname{ord}_{p}\left(\begin{array}{c}k+s / n \\ r\end{array}\right)$ or $\operatorname{ord}_{p}\left(\begin{array}{c}2 k-r-1 \\ j-r\end{array}\right)$ is positive for $j=k$ and $k-1$ and $0 \leqslant r \leqslant j$.

For $k \geqslant 10^{6}$, Lemma 3.1 will follow from application of Chebyshev-like estimates for primes, either in arithmetic progressions (due to Ramaré and Rumely [16]) or otherwise (due to Rosser and Schoenfeld [18] and Schoenfeld [20]), to the sets described in Lemma 3.2. The reader is directed to [6] for a more detailed account in the case when $n=3$. We illustrate the proof for $n=5$.

Suppose $n=5, s=1$ or 2 and $k \geqslant 10^{6}$. Lemma 3.2 implies that if $p \equiv$ $\pm s(\bmod 5)$ and $(k+1) / j \leqslant p \leqslant(5 k-6) /(5 j-1)$ for some positive integer $j \leqslant \sqrt{k / 5}$, then $p$ divides $G_{k}$. Similarly, if $p \equiv \pm 2 s(\bmod 5)$ and $(k+1) / j \leqslant p \leqslant$ $(5 k-6) /(5 j-2)$ for some $j \leqslant \sqrt{k / 5}$, then $p$ divides $G_{k}$. We apply Corollary $2^{*}$ and the closing remarks of $[\mathbf{2 0}]$ to derive a lower bound for the product of the primes in

$$
I_{1}=\bigcup_{j=1}^{[\sqrt{k / 5}]}\left[\frac{k+1}{j}, \frac{5 k-6}{5 j-1}\right]
$$

and then use Theorems 1 and 2 of [16] to do likewise for the primes $p \equiv \pm 2 s(\bmod 5)$ in

$$
I_{2}=\bigcup_{j=1}^{[\sqrt{k / 5}]}\left[\frac{5 k-6}{5 j-1}, \frac{5 k-6}{5 j-2}\right] .
$$

Regarding the first of these, if we further assume that $k<10^{9}$, then we can use the fact that

$$
\theta(x)=\sum_{p \leqslant x} \log p<x
$$

for $x<10^{11}$, to conclude (by applying Corollary $2^{*}$ of $[20]$ to the intervals $[(k+1) / j,(5 k-6) /(5 j-1)]$ for $1 \leqslant j \leqslant 24)$ that

$$
\sum_{p \in I_{1}} \log p>0.36496 k-4.96911 .
$$


Since $k<10^{9}$, we may apply the results of Table 2 of [16] to deduce that

$$
\sum_{\substack{p \in I_{2} \\ p \equiv \pm 2 s(\bmod 5)}} \log p>0 \cdot 24925 k-0 \cdot 29912
$$

whence

$$
\log G_{k}>0.61421 k-5 \cdot 26833>\log (1 \cdot 84) k .
$$

If we have $k \geqslant 10^{9}$, then we use the inequality $\theta(x)<1.000081 x$ (valid for $x>0$ ) and Theorem 1 of [16], in conjunction with the previously mentioned results, to reach the same conclusion.

To deal with the values of $k<10^{6}$, we argue as in the proof of Lemma 4.2 of [6]. Fixing $k$ and $s$, we explicitly compute the product of the primes in $I_{1}$, together with the primes congruent to $\pm 2 s(\bmod 5)$ in $I_{2}$. If we denote this product by $P_{k}$, we find that

$$
P_{k} / 1 \cdot 84^{k}>e^{-16}
$$

for all $1 \leqslant k<10^{6}$ and $1 \leqslant s \leqslant 2$ with exactly 154 exceptions, the largest being with $k=623$ and $s=2$. The arguments of Lemma 4.2 of [6] allow us to reduce this calculation from roughly two million values for $k$ to a few thousand. For the remaining pairs $(k, s)$ which fail to satisfy (7), we compute $G_{k}$ directly from the definition, verifying that

$$
G_{k} / 1 \cdot 84^{k}>e^{-16}
$$

in all cases (with $G_{k} / 1 \cdot 84^{k}$ minimal for $k=199$ and $s=2$ ).

We argue similarly for the other values of $n$, only with $e^{-16}$ replaced by $e^{-20}$ if $n=4$. In all cases, we apply the estimates of [16] and [20] to deduce bounds for $k \geqslant 10^{6}$ and then optimize the inequality for smaller $k$. All these computations were performed using Pari on Sparc IPC, Sparc 20 and Deck Alpha machines. In Table 2, we list the total number and largest exceptions to the inequality

$$
P_{k} / c_{1}(n)^{k}>c(n)
$$

for $1 \leqslant s<\frac{1}{2} n$, where $P_{k}$ is defined in the same way as before and $c(n)$ is $e^{-20}$ if $n=4$ and $e^{-16}$ otherwise. We also include the values of $k$ and $s$ which minimize $G_{k} / c_{1}(n)^{k}$.

\section{Proof of Theorems 1.1 and 1.3}

Define, for integral $k>0,0 \leqslant l, m \leqslant 1$ and $a, N, s$ and $n$ as in the hypotheses of either Theorem 1.1 or 1.3 ,

$$
p_{l m k}=N^{k} a^{2 k}\left(\prod_{p \mid n} p^{\max \left\{[k /(p-1)]+k \operatorname{ord}_{p}(n / a), 0\right\}}\right) G_{k}^{-1} p_{l m}(1 / N) .
$$

The results of the previous section, then, show that each $p_{l m k}$ is integral and we may apply Lemma 2.1 (Lemma 3.4 of [17] implies the non-vanishing of $\operatorname{det}\left(p_{\text {lmk }}\right)$ for each positive integer $k$ ). To prove Theorem 1.1 , we take $k_{0}=1$ and $t=3$ in Lemma 2.1, whence by Lemmas 2.2 and 3.1, we have, for $n \neq 4$,

$$
\left|\left(1+\frac{a}{N}\right)^{s / n}-\frac{p}{q}\right|>c^{-1}\left(\frac{3 \cdot 9(n-1)}{n} e^{16} q\right)^{-\lambda},
$$


TABLE 2.

\begin{tabular}{|c|c|c|c|}
\hline$n$ & exceptions to (8) & minimal & $\log \left(G_{k} / c_{1}(n)^{k}\right)$ \\
\hline 3 & $(k=3702, s=1)$ & $-13 \cdot 36$ & $(k=369, s=1)$ \\
\hline 4 & $352(k=3868, s=1)$ & $-19 \cdot 42$ & $(k=606, s=1)$ \\
\hline 5 & $154 \quad(k=623, s=2)$ & $-12 \cdot 65$ & $(k=199, s=2)$ \\
\hline 7 & $121 \quad(k=850, s=3)$ & $-13 \cdot 38$ & $(k=293, s=2)$ \\
\hline 11 & $111 \quad(k=467, s=2)$ & -12.43 & $(k=104, s=5)$ \\
\hline 13 & $(k=467, s=4)$ & -12.06 & $(k=257, s=2)$ \\
\hline 17 & $37 \quad(k=464, s=3)$ & $-8 \cdot 75$ & $(k=28, s=3)$ \\
\hline 19 & $(k=242, s=6)$ & -15.95 & $(k=74, s=6)$ \\
\hline 23 & $42 \quad(k=363, s=11)$ & $-12 \cdot 12$ & $(k=68, s=10)$ \\
\hline 29 & $(k=368, s=6)$ & $-10 \cdot 64$ & $(k=62, s=13)$ \\
\hline 31 & $(k=285, s=6)$ & $-11 \cdot 08$ & $(k=161, s=10)$ \\
\hline 37 & $(k=305, s=18)$ & $-10 \cdot 55$ & $(k=33, s=17)$ \\
\hline 41 & $(k=317, s=9)$ & -9.63 & $(k=152, s=7)$ \\
\hline 43 & $76(k=171, s=14)$ & $-9 \cdot 41$ & $(k=104, s=6)$ \\
\hline 47 & $(k=286, s=15)$ & $-8 \cdot 61$ & $(k=37, s=23)$ \\
\hline 53 & $(k=211, s=15)$ & $-10 \cdot 06$ & $(k=45, s=6)$ \\
\hline 59 & $(k=235, s=11)$ & -11.48 & $(k=75, s=26)$ \\
\hline 61 & $(k=362, s=13)$ & -7.64 & $(k=45, s=8)$ \\
\hline 67 & $(k=284, s=2)$ & $-7 \cdot 41$ & $(k=37, s=19)$ \\
\hline 71 & $671 \quad(k=283, s=19,34)$ & $-7 \cdot 46$ & $(k=36, s=19)$ \\
\hline
\end{tabular}

where

$$
c=\frac{n \kappa(a, n)}{(n-1) c_{1}(n)}(\sqrt{N}+\sqrt{N+a})^{2}
$$

with $\lambda$ and $c_{1}(n)$ as in Theorem 1.1. The bound $N / a \geqslant \frac{11}{5}$, derived from (4) and valid for all $n$, and the fact that $\lambda>2$, then, imply the stated result. For $n=4$ (whence $s=1$ ), we argue similarly only with the term $3 \cdot 9(n-1) / n$ replaced by 1.95 and $e^{16}$ replaced by $e^{20}$. It should be noted at this juncture that for specific $n$ in the range under consideration here, the constant $10^{10}$ appearing in the statement of Theorem 1.1 may be reduced through application of the precise minima given in Table 2. For instance, with $n=3$, we may replace $10^{10}$ by $3.23 \times 10^{6}$.

To prove Theorem 1.3 for $n \geqslant 14$, we use Lemma 2.1 with $k_{0}=1$ and $t=n /(n-5 \cdot 2)$ and set $a=s=1$. Bounding $G_{k}$ by the trivial estimate that $G_{k} \geqslant 1$ and noting that the constant 1.3 in Lemma 2.2(ii) may in fact be replaced by

$$
\sqrt{\frac{N+1}{N}}\left(2-\sqrt{\frac{N}{N+1}}\right)^{1 / n}
$$

yields Theorem 1.3 for these $n$, since

$$
\kappa(1, n)=n \mu_{n}
$$

To deal with the remaining values of $n$ requires a more detailed analysis. Specifically, we need a sharpened version of Lemma 2.2(i). Suppose that $k \geqslant 2$. We show that

$$
\left|I_{l}(1 / N)\right| \leqslant c\left(N(\sqrt{N}+\sqrt{N+1})^{2}\right)^{-k}
$$


where we may take $c=0.475$ if $n=3$ and $c=0.374$ if $4 \leqslant n \leqslant 13$. To prove this, we note, as in the proof of Lemma 2.2, that

$$
\left|I_{l}(1 / N)\right| \leqslant \frac{\left|1-e^{2 \pi i / n}\right|}{2 \pi} N^{-2 k} \int_{0}^{\infty} \frac{x^{k+1 / n} d x}{(x+1)((x+1)(x+1+1 / N))^{k}} .
$$

Explicitly computing

$$
\frac{\left|1-e^{2 \pi i / n}\right|}{2 \pi}\left(\frac{(\sqrt{N}+\sqrt{N+1})^{2}}{N}\right)^{k} \int_{0}^{\infty} \frac{x^{k+1 / n} d x}{(x+1)^{2 k+1}}
$$

for $3 \leqslant n \leqslant 13, N$ minimal satisfying the hypotheses of Theorem 1.3 and $2 \leqslant k \leqslant 25$, we find that this quantity is bounded above by either 0.475 (if $n=3$ ) or $0 \cdot 374$ (if $4 \leqslant n \leqslant 13$ ) in all cases, which proves (10) for $2 \leqslant k \leqslant 25$. To handle $k \geqslant 26$, we first use (11) to deduce the bound

$$
\left|I_{l}(1 / N)\right| \leqslant \frac{\left|1-e^{2 \pi i / n}\right|}{2 \pi} \frac{(n-1)^{(n-1) / n}}{n} N^{-2 k} \int_{0}^{\infty}\left(\frac{x}{(x+1)(x+1+1 / N)}\right)^{k} d x
$$

and evaluate this last integral in a similar fashion to Lemma 2.2, noting that it is bounded by the sum of

$$
\begin{gathered}
\int_{0}^{1 / 2}\left(\frac{x}{(x+1)^{2}}\right)^{k} d x \\
\int_{1 / 2}^{2}\left(\frac{x}{(x+1)(x+1+1 / N)}\right)^{k} d x
\end{gathered}
$$

and

$$
\int_{2}^{\infty}\left(\frac{x}{(x+1)^{2}}\right)^{k} d x
$$

This implies that

$$
\int_{0}^{\infty}\left(\frac{x}{(x+1)(x+1+1 / N)}\right)^{k} d x
$$

is bounded above by

$$
\frac{3}{2}\left(\frac{N}{(\sqrt{N}+\sqrt{N+1})^{2}}\right)^{k}+\frac{1}{2}\left(\frac{2}{9}\right)^{k}+\sum_{r=2}^{\infty}\left(\frac{r}{(r+1)^{2}}\right)^{k} .
$$

Since the hypotheses of Theorem 1.3 for the values of $n$ in question force $N \geqslant 6$, we have

$$
\frac{(\sqrt{N}+\sqrt{N+1})^{2}}{N} \leqslant 4 \cdot 327
$$

so that $k \geqslant 26$ yields

$$
\int_{0}^{\infty}\left(\frac{x}{(x+1)(x+1+1 / N)}\right)^{k} d x \leqslant 2 \cdot 5\left(\frac{N}{(\sqrt{N}+\sqrt{N+1})^{2}}\right)^{k} .
$$

Since further

$$
\frac{\left|1-e^{2 \pi i / n}\right|}{2 \pi} \frac{(n-1)^{(n-1) / n}}{n} \leqslant 0 \cdot 146
$$


for $3 \leqslant n \leqslant 13$, we have (10) as desired for these $k$ also (with $c=0.475$ for $n=3$ or $c=0.374$ for $4 \leqslant n \leqslant 13$ ). If we replace 1.3 in Lemma 2.2(ii) by (9), this yields Theorem 1.3 for $3 \leqslant n \leqslant 13$ with

$$
q \geqslant \frac{(\sqrt{N}+\sqrt{N+1})^{2}}{n \mu_{n}}
$$

through application of Lemma 2.1 with $t=1.905(n=3)$ or $t=1.598(4 \leqslant n \leqslant 13)$ and $k_{0}=2$ (where, again, we use the lower bounds upon $N$ derived from the hypotheses of Theorem 1.3).

To complete the proof of Theorem 1.3, we need to show that the desired inequality holds for all

$$
q<\frac{(\sqrt{N}+\sqrt{N+1})^{2}}{n \mu_{n}}
$$

with $3 \leqslant n \leqslant 13$ and $N$ as previously. If $p=q=1$, then the result obtains from the mean value theorem, so we may assume that $p>q \geqslant 1$. If $q$ satisfies (13), then

$$
\frac{p}{q} \geqslant \frac{q+1}{q}>1+\frac{n \mu_{n}}{(\sqrt{N}+\sqrt{N+1})^{2}}
$$

and the mean value theorem yields

$$
\sqrt[n]{1+\frac{1}{N}}<1+\frac{1}{n N}
$$

so that

$$
\left|\sqrt[n]{1+\frac{1}{N}}-\frac{p}{q}\right|>\frac{n \mu_{n}}{(\sqrt{N}+\sqrt{N+1})^{2}}-\frac{1}{n N} .
$$

By (12), we therefore have

$$
\left|\sqrt[n]{1+\frac{1}{N}}-\frac{p}{q}\right|>\frac{n}{4 \cdot 237 N}-\frac{1}{n N}>\frac{1}{3 N},
$$

which easily implies Theorem 1.3.

\section{Proof of Corollary 1.2}

We will illustrate the proof of Corollary 1.2 with the example $\theta=\sqrt[4]{5}$. Here we take $N=80$ and $a=1$ in Theorem 1.1 and may thus conclude that

$$
\left|\sqrt[4]{81 / 80}-\frac{3 q}{2 p}\right|>80^{-1}\left(2 \times 10^{10} p\right)^{-\lambda}
$$

for

$$
\lambda=1+\frac{\log \left((8 / 1 \cdot 62)(4 \sqrt{5}+9)^{2}\right)}{\log \left((1 \cdot 62 / 8)(4 \sqrt{5}+9)^{2}\right)}=2 \cdot 76457 \ldots
$$


and any integers $p$ and $q$ (with $q \neq 0$ ). It follows that

$$
\left|\sqrt[4]{5}-\frac{p}{q}\right|>\sqrt[4]{5}\left(\frac{p}{q}\right)^{1-\lambda} 120^{-1}\left(2 \times 10^{10} q\right)^{-\lambda}
$$

whence

$$
\left|\sqrt[4]{5}-\frac{p}{q}\right|>\left(4.906267 \times 10^{30}\right)^{-1} q^{-2 \cdot 764575}
$$

holds for all non-zero $p$ and $q$. This implies that

$$
\left|\sqrt[4]{5}-\frac{p}{q}\right|>0 \cdot 03 q^{-2 \cdot 77}
$$

provided $q \geqslant 10^{5377}$. To deal with $q$ satisfying $1 \leqslant q<10^{5377}$, we note that a rational $p / q$ which fails to satisfy (14) must be a convergent in the continued fraction expansion to $\sqrt[4]{5}$, say $p_{i} / q_{i}$. We therefore have

$$
\left|\sqrt[4]{5}-\frac{p_{i}}{q_{i}}\right|>\frac{1}{\left(a_{i+1}+2\right) q_{i}^{2}}
$$

where $a_{i+1}$ is the $(i+1)$ th partial quotient in this continued fraction expansion (see for example, $[\mathbf{1 5}]$ ). This implies that

$$
a_{i+1}>33 q_{i}^{0 \cdot 77}-2 .
$$

Checking that the first forty convergents to $\sqrt[4]{5}$ satisfy (14) and noting that $q_{41}>10^{21}$, implies, from (15), that we need only show that $a_{j}<10^{17}$ for $j<12000$ (since we verify that $q_{12000}>10^{6000}$ ). Computing the desired partial quotients via Pari (gp), we find that the largest of the first 12000 partial quotients is equal to 4057 , which enables us to conclude as desired.

Similar arguments apply for our other examples. The bounds in Corollary 1.2 follow from Theorem 1.1 with the choices of $a, N, s$ and $n$ noted in Table 3, provided, in each case, $q<10^{6000}$. Computing the first 12000 partial quotients for each $\theta$ and the related convergents, we list the largest partial quotients in this range. In every case, the 12000th convergent in the continued fraction expansion to $\sqrt[n]{m}$ has denominator exceeding $10^{6000}$ and analogous bounds to (15) enable us to conclude as in Corollary 1.2.

\section{Some Diophantine consequences}

In [7], the author (joint with B. M. M. de Weger) applies these techniques, together with lower bounds for linear forms in logarithms of algebraic numbers, to the problem of bounding the number of solutions to the binomial Thue equation $\left|a x^{n}-b y^{n}\right|=1$. For special cases of this and related equations, Corollary 1.2, via the factorization

$$
x^{n}-m y^{n}=(x-\sqrt[n]{m} y)\left(x^{n-1}+\sqrt[n]{m} x^{n-2} y+\ldots+m^{(n-1) / n} y^{n-1}\right)
$$

provides a means for determining all solutions explicitly. By way of example, for the equations considered in $\S 5$ of [11], we have 
TABLE 3.

\begin{tabular}{ccc|ccc}
\hline$\theta$ & $s, n, a, N$ & $\max a_{i}$ & $\theta$ & $s, n, a, N$ & $\max a_{i}$ \\
\hline$\sqrt[4]{5}$ & $1,4,1,80$ & 4057 & $\sqrt[5]{34}$ & $1,5,1,16$ & 61217 \\
$\sqrt[4]{14}$ & $1,4,1,7$ & 16353 & $\sqrt[5]{37}$ & $1,5,5,32$ & 91267 \\
$\sqrt[4]{15}$ & $1,4,1,15$ & 38166 & $\sqrt[5]{39}$ & $2,5,10,3^{7}$ & 37108 \\
$\sqrt[4]{17}$ & $1,4,1,16$ & 5301 & $\sqrt[5]{40}$ & $1,5,1,4$ & 6720 \\
$\sqrt[4]{18}$ & $1,4,1,8$ & 284610 & $\sqrt[5]{42}$ & $1,5,5,16$ & 23743 \\
$\sqrt[4]{37}$ & $1,4,28,15^{4}$ & 48324 & $\sqrt[7]{5}$ & $2,7,3,125$ & 58199 \\
$\sqrt[4]{39}$ & $1,4,1,624$ & 8611 & $\sqrt[7]{10}$ & $3,7,7,25$ & 180016 \\
$\sqrt[5]{3}$ & $2,5,5,27$ & 21171 & $\sqrt[7]{11}$ & $3,7,7,121$ & 76362 \\
$\sqrt[5]{6}$ & $2,5,1,8$ & 16724 & $\sqrt[7]{12}$ & $3,7,1,8$ & 6793 \\
$\sqrt[5]{10}$ & $2,5,3,125$ & 8835 & $\sqrt[7]{13}$ & $2,7,10,37$ & 31257 \\
$\sqrt[5]{11}$ & $1,5,122,21^{5}$ & 266282 & $\sqrt[7]{17}$ & $1,7,11,2176$ & 9333 \\
$\sqrt[5]{15}$ & $2,5,2,25$ & 64273 & $\sqrt[7]{23}$ & $2,7,95,23^{4}$ & 47970 \\
$\sqrt[5]{18}$ & $2,5,1,3$ & 19358 & $\sqrt[7]{45}$ & $3,7,2,25$ & 10659 \\
$\sqrt[5]{22}$ & $1,5,5,11$ & 295784 & $\sqrt[11]{48}$ & $5,11,1,8$ & 26741 \\
$\sqrt[5]{28}$ & $1,5,1,7$ & 7117 & $\sqrt[13]{6}$ & $5,13,13,243$ & 66846 \\
$\sqrt[5]{30}$ & $1,5,1,15$ & 7812 & $\sqrt[11]{20}$ & $4,13,3,125$ & 8300 \\
$\sqrt[5]{31}$ & $1,5,1,31$ & 45545 & $\sqrt[17]{50}$ & $5,17,3,125$ & 4130182 \\
$\sqrt[5]{33}$ & $1,5,1,32$ & 22143 & & & \\
\hline
\end{tabular}

COROllary 6.1. If $x$ and $y$ are integers, then

$$
\begin{aligned}
\left|x^{5}-3 y^{5}\right| & \geqslant(\max \{|x|,|y|\})^{1 \cdot 34}, \\
\left|x^{7}-5 y^{7}\right| & \geqslant(\max \{|x|,|y|\})^{2 \cdot 57}, \\
\left|x^{7}-13 y^{7}\right| & \geqslant(\max \{|x|,|y|\})^{2 \cdot 09},
\end{aligned}
$$

and

$$
\left|x^{7}-17 y^{7}\right| \geqslant(\max \{|x|,|y|\})^{1 \cdot 80} .
$$

It is easy to derive analogous bounds for the other examples in Corollary 1.2. In general (see [4] and [6] for the cubic situation), when the Padé approximation machinery of Theorem 1.1 may be applied to deduce an irrationality measure for a particular $\sqrt[n]{m}$, the resulting measure will almost certainly be stronger than that obtained via the theory of linear forms in logarithms. On the other hand, the latter theory applies for all algebraic numbers, while, arguing as in [6], if one defines $N_{n}(x)$ to be the number of positive integers less than or equal to $x$ for which Theorem 1.1 may be applied to produce a non-trivial effective measure of irrationality, one has only

$$
N_{n}(x) \gg x^{(5 n-8) / 2 n(n-1)} .
$$

The arithmetic 'flukes' that enable one to appeal to Theorem 1.1 correspond to exceptional convergents in the continued fraction expansion to $\sqrt[n]{m}$. As noted in [10], these in turn are related to extreme examples in the $a b c$-conjecture. 


\section{Concluding remarks}

The fundamental improvement over [1] and [2] that one finds in $[\mathbf{6}, \mathbf{1 1}, \mathbf{1 2}, \mathbf{1 4}]$ and this paper obtains from lower bounds upon the quantity $G_{k}=G_{k}(n)$ defined in $\S 3$ (or related quantities in [11] and [12]). While asymptotically, our approach and that of [11] are equivalent (see [5]), for approximation to algebraic numbers of degrees 3, 4 and 6, we are able to avoid reference to primes in arithmetic progressions, leading to sharper explicit results. In fact (again, see [5]), for any $n \geqslant 3$ one can show that

$$
\lim _{k \rightarrow \infty} \frac{1}{k} \log \left(G_{k}(n)\right)=-\gamma-\frac{2}{\phi(n)} \sum_{\substack{1 \leq r<n / 2 \\(r, n)=1}} \Psi\left(\frac{n-r}{n}\right),
$$

where $\gamma$ is the Euler-Mascheroni constant, $\phi(n)$ is Euler's totient function and $\Psi(z)$ is the derivative of the logarithm of $\Gamma(z)$. For $n=3$, then, we have

$$
\lim _{k \rightarrow \infty} G_{k}(3)^{1 / k}=3 \sqrt{3} e^{-\pi / 2 \sqrt{3}}=2 \cdot 09807 \ldots
$$

which compares quite well with our lower bound given in Theorem 1.1 of $c_{1}(3)=2 \cdot 03$. Denoting $\lim _{k \rightarrow \infty} G_{k}(n)^{1 / k}$ by $\alpha(n)$, via Euler-Maclaurin summation, one can show that

$$
\lim _{n \rightarrow \infty} \alpha(n)=\pi / e^{\gamma}=1.76387 \ldots
$$

and even that $\alpha(n)>\pi / e^{\gamma}$ if $p$ is prime. A comparison to Theorem 1.1 shows that for larger $n$ (where we use the bounds for primes in arithmetic progressions due to Ramaré and Rumely [16]), we are increasingly unable to approach the asymptotic results in strength. Improvements in these latter estimates would therefore be of clear interest in this regard.

\section{References}

1. A. BAKER, 'Rational approximations to certain algebraic numbers', Proc. London Math. Soc. (3) 14 (1964) 385-398.

2. A. BAKER, 'Rational approximations to $\sqrt[3]{2}$ and other algebraic numbers', Quart. J. Math. Oxford Ser. (2) 15 (1964) 375-383.

3. A. BAKER, 'The theory of linear forms in logarithms', Transcendence theory: advances and applications (ed. A. Baker, Academic Press, London, 1977), pp. 1-27.

4. A. BAKER and C. L. STEWART, 'On effective approximation to cubic irrationals', New advances in transcendence theory (ed. A. Baker, Cambridge University Press, 1988), pp. 1-24.

5. M. A. BENNETT, 'Simultaneous rational approximation to binomial functions', Trans. Amer. Math. Soc. 348 (1996) 1717-1738.

6. M. A. BenNETT, 'Effective measures of irrationality for certain algebraic numbers', J. Austral. Math. Soc. to appear.

7. M. A. BenNetT and B. M. M. DE Weger, 'On the Diophantine equation $\left|a x^{n}-b y^{n}\right|=1$ ', Math. Comp. to appear.

8. E. Bombieri, 'On the Thue-Siegel-Dyson theorem', Acta Math. 148 (1982) 255-296.

9. E. Bombieri and J. MUELLER, 'On effective measures of irrationality for $(a / b)^{1 / r}$ and related numbers', J. Reine Angew. Math. 342 (1983) 173-196.

10. J. BRowkin and J. BRZEZINŚKI, 'Some remarks on the abc-conjecture', Math. Comp. 62 (1994) 931-939.

11. G. V. Chudnovsky, 'On the method of Thue-Siegel', Ann. of Math. (2) 117 (1983) 325-382.

12. D. EASTON, 'Effective irrationality measures for certain algebraic numbers', Math. Comp. 46 (1986) 613-622.

13. N. I. Fel'Dman, 'An effective refinement of the exponent in Liouville's theorem', Math. USSR-Izv. 5 (1971) 985-1002.

14. A. Heimonen, T. Matala-AhO, and K. VÄÄnÄNEN, 'An application of Jacobi type polynomials to irrationality measures', Bull. Austral. Math. Soc. 50 (1994) 225-243. 
15. W. J. LeVeque, Topics in number theory (Addison-Wesley, Reading, Mass., 1956).

16. O. Ramaré and R. Rumely, 'Primes in arithmetic progressions', Math. Comp. 65 (1996) 397-425.

17. J. H. RICKERT, 'Simultaneous rational approximations and related diophantine equations', Math. Proc. Cambridge Philos. Soc. 113 (1993) 461-472.

18. J. Rosser and L. SCHOENFELD, 'Sharper bounds for the Chebyshev functions $\theta(x)$ and $\psi(x)$ ', Math. Comp. 29 (1975) 243-269.

19. K. F. Rотн, 'Rational approximations to algebraic numbers', Mathematika 2 (1955) 1-20.

20. L. SCHOENFELD, 'Sharper bounds for the Chebyshev functions $\theta(x)$ and $\psi(x)$ II', Math. Comp. 30 (1976) 337-360.

Department of Mathematics

University of Michigan

Ann Arbor

Michigan 48109

U.S.A.

E-mail: mabennet@math.lsa.umich.edu 